\title{
Empagliflozin senkt Herzinsuffizienz-Risiko um ein Drittel
}

\author{
Einer neuen Auswertung der EMPA-REG-Studie zufolge reduziert der SGLT2-Inhibitor \\ Empagliflozin bei kardiovaskulär erkrankten Diabetikern auch das Herzinsuffizienz-Risiko \\ schnell, deutlich und unabhängig davon, ob bereits eine Herzschwäche vorliegt.
}

\begin{abstract}
Die EMPA-REG OUTCOME-Studie ist aufgrund der praktischen Konsequenzen wohl eine der bedeutendsten Untersuchungen, welche die Diabetologie in den letzten zehn Jahren hervorgebracht hat. Die im September auf dem europäischen Diabetologenkongress in Stockholm publizierten Ergebnisse zeigten erstmals überzeugend, dass eine antidiabetisch wirksame Substanz kardiovaskuläre Komplikationen verhindert und die Prognose verbessert. Die relative Risikosenkung für die Gesamtmortalität unter Empagliflozin betrug 32\%. Dies ist noch günstiger als die Therapie von kardiovaskulären Hochrisikopatienten mit Statinen oder mit ACE-Hemmern in der $4 S$ Studie bzw. in der HOPE-Studie.

Beim AHA-Jahreskongress 2015 in Orlando berichtete nun EMPA-REGStudienautor Silvio E. Inzucchi von der Yale University School of Medicine in New Haven neue Daten der Studie.
\end{abstract}

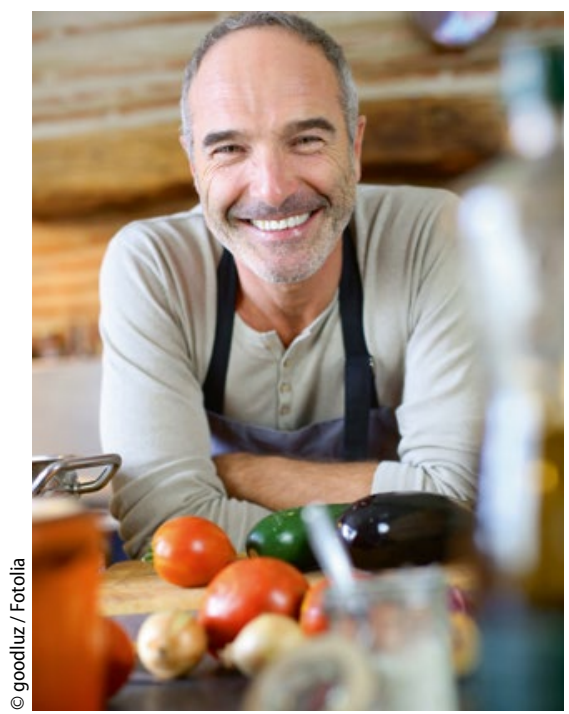

Keine Wirkung auf Herzinfarkt und Schlaganfall, ...

Primärer Endpunkt der Studie war die Kombination Herztod, Herzinfarkt und Schlaganfall. Dieses Risiko wurde durch Empagliflozin von $12,1 \%$ auf $10,5 \%$ absolut um $1,6 \%$ und relativ um $14 \%$ gesenkt $(\mathrm{p}=0,04)$. Verantwortlich für den Unterschied war der härteste der drei Einzelendpunkte, die kardiovaskuläre Mortalität, die von 5,9\% auf 3,7\% zurückging (HR: 0,62). Keinen Einfluss hatte die Therapie auf Herzinfarkt oder Schlaganfall.

... aber auf das Herzinsuffizienz-Risiko!

Das Risiko für die Entwicklung einer Herzinsuffizienz wurde erheblich reduziert: Das relative Risiko für eine Krankenhauseinweisung wegen Herzinsuffizienz lag im Vergleich zur Kontrollgruppe um $35 \%$ niedriger $(p=0,0017)$, dasjenige für Krankenhauseinweisung oder Tod aufgrund von Herzinsuffizienz um
$39 \%(p=0,0002)$. Der Effekt stellte sich früh im Studienverlauf ein.

In der Minderheit der Patienten, die schon zu Studienbeginn eine Herzinsuffizienz aufwiesen, betrug das Risiko für erneute Klinikeinweisungen wegen Herzschwäche oder Herztod unter Empagliflozin 16,2\% und in der Kontrollgruppe 20,1\%, wobei der Unterschied aufgrund der kleinen Fallzahl nicht signifikant war.

In der viel größeren Gruppe der Patienten ohne Herzinsuffizienz zu Beginn wurden 4,5\% (Empagliflozin) bzw. 7,1\% (Kontrollen) wegen Herzinsuffizienz eingewiesen oder verstarben - eine statistisch signifikante Risikoreduktion um $37 \%$.

Dr. med. Dirk Einecke

- Quelle: American Heart Association, Scientific Sessions 2015, Orlando, 07.-11. November 2015; Zinman B, et al.; Empagliflozin, Cardiovascular Outcomes, and Mortality in Type 2 Diabetes. NEngl J Med 2015; doi: 10.1056/NEJMOA1504720

\section{Fertiggerichte sind ungesund}

\section{Hausmannskost schützt vor Diabetes}

\begin{abstract}
Selbst zubereitete und zuhause verzehrte Mahlzeiten schützen vor der Entwicklung von Übergewicht und Diabetes, berichten Autoren aus Boston. Sie hatten Daten von annähernd $100.000 \mathrm{im}$ Gesundheitswesen Beschäftigen ausgewertet, die bis zu 36 Jahre lang nachverfolgt wurden. Sie fanden dabei eine lineare Beziehung zwischen nicht selbst zubereiteten Mahlzeiten und dem Diabetes-Risiko. Seit Jahrzehnten gebe es einen Trend zu kommerziell vorgefertigten Fertiggerichten niedriger Qualität.
\end{abstract}

Wer 11-14 Mahlzeiten pro Woche zuhause aß, hatte ein um $13 \%$ geringeres Risiko als Personen, die 0 bis 6-mal pro Woche zuhause speisten. Die Autoren berichten, dass Abendessen außer Haus das Diabetesrisiko besonders deutlich erhöhten. Personen, die viele Mahlzeiten selbst zubereiteten, verzehrten mehr Obst, Gemüse, Vollkornprodukte und rotes Fleisch.

- American Heart Association, Scientific Sessions 2015, Orlando 07.-11. November 2015 